\title{
Finite-temperature properties of nonmagnetic transition metals: Comparison of the performance of constraint-based semilocal and nonlocal functionals
}

\author{
Leili Gharaee and Paul Erhart* \\ Chalmers University of Technology, Department of Physics, Gothenburg, Sweden \\ Per Hyldgaard ${ }^{\dagger}$ \\ Chalmers University of Technology, Department of Microtechnology and Nanoscience, MC2, Gothenburg, Sweden
}

(Received 2 December 2016; published 27 February 2017)

\begin{abstract}
We assess the performance of nonempirical, truly nonlocal, and semilocal functionals with regard to structural and thermal properties of $3 d, 4 d$, and $5 d$ nonmagnetic transition metals. We focus on constraint- based functionals and consider the consistent-exchange van der Waals density-functional version vdW-DF-cx [Phys. Rev. B 89, 035412 (2014)], the semilocal PBE functional [Phys. Rev. Lett. 77, 3865 (1996)], and the PBEsol functional [Phys. Rev. Lett. 100, 136406 (2008)], as well as the AM05 metafunctional [Phys. Rev. B 72, 085108 (2005)]. Using the quasiharmonic approximation, the structural parameters, elastic response, and thermal expansion at finite temperatures are computed and compared to experimental data. We also compute cohesive energies explicitly including zero-point vibrations. It is shown that overall vdW-DF-cx provides an accurate description of thermal properties and retains a level of transferability and accuracy that is comparable to or better than some of the best constraint-based semilocal functionals. Especially, with regard to the cohesive energies, the consistent inclusion of spin-polarization effects in the atoms turns out to be crucial, and it is important to use the rigorous spin-vdW-DF-cx formulation [Phys. Rev. Lett. 115, 136402 (2015)]. This demonstrates that vdW-DF-cx has general-purpose character and can be used to study systems that have both sparse and dense electron distributions.
\end{abstract}

DOI: 10.1103/PhysRevB.95.085147

\section{INTRODUCTION}

The adiabatic connection formula (ACF) enables a formal determination of all exchange-correlation (XC) effects in density-functional theory (DFT) [1-3]. The XC energy density functional (DF) $E_{\mathrm{xc}}$ can be seen as the electrostatic binding of electrons with its associated XC hole. Good $E_{\mathrm{xc}}$ approximations represent the core of DFT, and $E_{\mathrm{xc}}$ formulations reflect insight pertaining to the collective response of the interacting electron gas [2,3]. Important progress was achieved by enforcing hole conservation and other physical constraints in the formulation of the local density approximation (LDA) as well as semilocal functionals based on the generalized gradient approximation (GGA). Hole conservation underpins, for example, the PBE functional [4], that has proven to be highly successful as a general-purpose functional for problems in which physical behavior is governed by the response at large electron concentrations. Specifically, the PBE functional is accurate for both hard materials and individual molecules, and accordingly it has found widespread applications [5].

The past decade has witnessed the successful introduction of the van der Waals (vdW) density-functional (vdW-DF) method [6-10], launched as a systematic extension [11-13] of the LDA and the GGA. Unlike local and semilocal functionals such as the latter two, it describes also the much larger class of sparse systems [14], e.g., molecular solids, layered materials, and weak chemisorption cases, in which binding across

\footnotetext{
*erhart@chalmers.se

†hyldgaar@chalmers.se
}

internal voids arises from a truly nonlocal, vdW-type binding. The Chalmers-Rutgers vdW-DF method is focused on the electron response and has both regular releases (vdW-DF1 [7], vdW-DF2 [15], and vdW-DF-cx [16]) and variants (including vdW-DF-C09 [17], optB88, optPBE [18], optB86b [19], and rev-vdW-DF2 [20]). It has recently been extended with a rigorous spin formulation that reflects the vdW-DF design logic [21]. There are also related but alternative formulations of the nonlocal-correlation term in the Vydrov-van Voorhis family (VV09 [22], vdW-DF-09 [23], and VV10 [24]) and approaches that emphasize multipole response and mutual coupling of exchange holes [25-27]. In addition, there are approaches that focus on the dipole and multipole response of orbitals, atoms, and clusters (typically obtained outside of the ground-state DFT framework), compute the mutual coupling energy, and add it to a traditional ground-state DFT description [28-39]. It is commonly required that the chosen approach must be accurate both when there are important regions of low-electron concentration, i.e., sparse matter such as in intermolecular binding [14], and when studying dense matter, i.e., harder materials such as transition metals.

The recent consistent-exchange version, vdW-DFcx $[9,13,16]$, uses the vdW-DF plasmon-pole response description also to determine the semilocal component of the $\mathrm{vdW}-\mathrm{DF}$ method. vdW-DF-cx is determined by a dielectric-response description that automatically enforces current conservation in the screening response [7,13]. The vdW-DF-cx method effectively uses the same plasmon-pole response to define both gradient-corrected exchange and nonlocal correlations. This new vdW-DF version thereby minimizes a hidden crossover term $\delta E_{x}^{0}$ that generally enters in the vdW-DF family of functionals [16]. 
vdW-DF-cx has already proven itself accurate and useful in a number of problems that involve both regions of sparse and dense electron distributions such as molecular dimers $[16,40]$, layered materials [9,41-45], semiconductors [9,46], molecular crystals [47,48], adsorption processes [21,49], as well as weak chemisorption, molecular switching, and molecular selfassembly $[21,49,50]$. The ACF foundation and the emphasis on conservation laws in the vdW-DF-cx construction further suggests a general-purpose nature [9] and motivates a comprehensive investigation of its performance also for regular dense matter. This is particularly interesting since earlier members of the vdW-DF family have repeatedly been found to yield an inferior description of traditional bulk materials, in particular the late transition metals [49].

Here, we benchmark vdW-DF-cx for thermophysical properties of nonmagnetic transition metals, for which extensive experimental data are available for comparison [51-53]. Specifically, we consider lattice parameters, thermal expansion, and bulk moduli at finite temperature as well as the cohesive energies including zero-point contributions at the level of the quasiharmonic approximation (QHA). This data set explicitly tests not only the description of energy and structure but also forces, going beyond the set of properties commonly considered in comparative assessments of $\mathrm{XC}$ functionals. In addition to vdW-DF-cx, we consider the constraint-based semilocal functionals, PBE [4] and PBEsol [54], as well as AM05 [55].

We show that vdW-DF-cx meets and exceeds the performance of the PBE, PBEsol, and AM05 functionals for nonmagnetic transition metals. This suggests that vdW-DF-cx provides a good, balanced description of nonlocal exchange and nonlocal correlation also in these types of materials. $\mathrm{vdW}-\mathrm{DF}-\mathrm{cx}$ thus remains a candidate for serving as a generalpurpose materials-theory tool, working for both hard and soft matter [9].

The remainder of this paper is organized as follows. The next section provides an overview of the constraintbased functionals considered in the present work, while methodological aspects are compiled in Sec. III A. Section IV describes the main results, and Sec. $\mathrm{V}$ provides a summary and conclusions. A detailed compilation of results including a per-element comparison with experimental data can be found in the supplementary material [56].

\section{CONSTRAINT-BASED NONLOCAL FUNCTIONALS}

\section{A. General aspects}

Comparisons among constraint-based nonlocal functionals are valuable in our drive to further improve truly nonlocal DFs. Several previous studies have shown that some vdW-DF approaches can work well for solids [9,18-20,39,57]. While this has helped build trust in the vdW-DF method, constraintbased functionals such as vdW-DF-cx, PBE, and PBEsol are all linked to the ACF and conservation [4,13,16,54] and can thus be expected to yield good transferability. Similarly, AM05 is linked to other constraints, interpolating between model systems [54,55]. Yet as briefly reviewed below, different physical aspects were emphasized in their construction, and one can thus expect to gain insight into strengths and limitations of each approach exactly because these functionals are each representatives of a specific design logic. By focusing the present benchmark on constraint-based functionals, we are thus able to draw more general conclusions.

The four functionals considered in the present work share some common traits while also having some distinct differences in their design logic, making it interesting to contrast their performance. All matter has internal surfaces with a variation between higher and lower electron density regions, and insight from surface physics underpins all the designs. It led Langreth and Perdew to the early GGA [3] and it entered the specification of gradient-corrected correlation in PBEsol [54] and AM05 [55]. These concepts are also central to the development of the vdW-DF method [6,10], which takes the surface idea further than in the GGA. This is done by noting that a semilocal representation of the electron-gas response does not retain a full description of the electrodynamical coupling among (GGA) XC holes [6,10-13]. The electrodynamical coupling is relevant, for example, when there are multiple interacting density fragments (molecules or surfaces) separated by a region with low electron concentration $[6,13]$.

The Fermi wave vector $k_{F}(\boldsymbol{r})=\left[3 \pi^{2} n(\boldsymbol{r})\right]^{1 / 3}$ sets a local energy scale via the LDA exchange energy per particle, $\varepsilon_{x}^{\mathrm{LDA}}(\boldsymbol{r})=-(3 / 4 \pi) k_{F}(\boldsymbol{r})$. We take semilocal functionals to imply that the XC hole form or the energy per particle depend exclusively on the local (spin) density $n(\boldsymbol{r})$ and the local scaled gradient $s(\boldsymbol{r})=|\nabla n| /\left[2 n(\boldsymbol{r}) k_{F}(\boldsymbol{r})\right]$. Semilocal GGA functionals can be expressed via the local variation in the $\mathrm{XC}$ energy per particle,

$$
\varepsilon_{\mathrm{xc}}^{\mathrm{GGA}}(\boldsymbol{r}) \equiv F_{\mathrm{xc}}(n(\boldsymbol{r}), s(\boldsymbol{r})) \varepsilon_{x}^{\mathrm{LDA}}(\boldsymbol{r})
$$

Here, the XC enhancement factor $F_{\mathrm{xc}}(n(\boldsymbol{r}), s(\boldsymbol{r}))$ reflects the physical nature of the associated semilocal XC hole [4,54].

In addition to functionals that (like PBE, PBEsol, AM05, and present vdW-DF versions) use a gradient-corrected exchange description, there are also ongoing developments of constraint-based meta-GGAs (MGGAs), including the new SCAN-MGGA and TM-MGGA [58-60]. These functionals also employ the variation in the local kinetic-energy density to refine the account of nonlocal XC effects. The inclusion of such higher-order XC effects is important for a description of weaker binding at intermediate distances, and it also improves the description of the bulk structure and cohesion over that of the constraint-based GGAs [59,60]; not considering dispersive interactions, the performance of such new MGGAs for bulk structure (as obtained in other codes) is better than what we are documenting here for vdW-DF-cx [59]. It is, however, beyond the present scope to also benchmark these new highly constraint-based MGGAs.

In a continued development of the vdW-DF method, we chose to focus on constraints. There are at least four important criteria that can help the design of robust and transferable density functionals:

(i) Conservation of the exchange-correlation $(\mathrm{XC})$ hole [2,3,9,13,61-63].

(ii) Current conservation in the description of the electrodynamical response that underpins the definition of the $\mathrm{XC}$ hole via the ACF $[2,3,13,64]$. 
(iii) Adherence to the global Lieb-Oxford bound ( $\mathrm{g}-\mathrm{LOB}$ ) [65-67].

(iv) Most relevant for developing the vdW-DF method: avoidance of spurious exchange (non-vdW) binding arising at intermediate-to-far fragment separations [7,68,69].

Charge conservation, criterion (i), has long been understood as being essential for the design of robust, transferable density functionals. After all, the combination of the electron and its associated XC hole must be charge-neutral for the DFT treatment (as a noninteracting quasiparticle) to be sensible [3]. Compliance with criterion (ii) is essential in the definition of the vdW-DF method $[7,10]$, and it was explicitly used in motivating the formulation of the vdW-DF-cx method [13]. Also, closely related insight on the $F$-sum rule was central in the definition of both the LDA $[2,3,64]$ and the constraint-based GGAs (r)PW86 [69,70], PBE [4], and PBEsol [54]. Criterion (iv) is relevant for avoiding double counting in the present vdW-DF versions (which starts with gradient-corrected exchange [13]). Seeking a meta-vdW-DF (starting instead from the new MGGAs) is interesting since it may thus be possible to better discriminate between spurious and physical binding at intermediate distances $[59,60]$; at present, however, one is motivated to pick the exchange enhancement $F_{x}(s) \sim s^{2 / 5}$ asymptotically $[6,7,10,15,69]$. The original and revised PW86 [69,70], PBE [4], PBEsol [54], as well as both the original vdW-DF and the new vdW-DF-cx should be seen as highly constrained: They incorporate the first two criteria [(i) and (ii)], while criteria (iii) and (iv) are expected to be fulfilled in actual calculations.

\section{B. The PBE functional}

The PBE functional [4] is an important example of a constraint-based GGA [5,71]. The PBE was designed by first constructing a numerical GGA with an enhancement factor $\tilde{F}_{\mathrm{xc}}$ that reflects conditions on the shape of the semilocal XC hole description and, in a subsequent step, by extracting an analytical form for the PBE $F_{\mathrm{xc}}$ for practical use. One can expect a high degree of transferability because it is anchored in conservation laws [2,3]. In fact, the PBE functional has had a huge impact on materials theory and has turned out to be an extremely successful general-purpose functional for systems with dense electron distributions including both individual molecules and hard materials [5].

\section{The PBEsol functional}

One of the best-performing constraint-based semilocal functionals for condensed matter is the PBEsol functional [54]. While, as in the case of PBE, the nature of screened manybody response and the $\mathrm{XC}$ hole were emphasized during its construction, its authors also relied on other formal results in its design. The GGA framework that underpins both PBE and PBEsol is very powerful, but it is not possible to satisfy all constraints at the same time. ${ }^{1}$ The PBE functional is highly transferable and works very well for both molecular formation

\footnotetext{
${ }^{1}$ The relation between PBE and PBEsol reflects in part the physical insight used for picking the form of gradient-corrected exchange enhancements [54]. Both functionals use conserving (albeit different)
}

energies and the structure and energies of hard materials. As a result of the diagrammatic (gradient-expansion) emphasis, PBEsol yields an even better description of the structure of hard materials [54].

PBE and PBEsol are designed also to comply with a socalled local Lieb-Oxford bound (1-LOB). This condition is formulated as

$$
F_{x}(s(r))<1.804 \text {. }
$$

By complying to Eq. (2), the g-LOB criterion (3) is automatically satisfied. It should also be noted that there exist densities (shell-like structure) for which breaking the l-LOB also means violating the $\mathrm{g}$-LOB $[65,67]$. It is desirable to explicitly test compliance with the g-LOB criterion (3) for actual calculations when the GGA-exchange description can violate the 1-LOB condition (2).

\section{The AM05 functional}

The AM05 constraint-based functional [55] performs very well for describing the structure of dense materials. The AM05 provides, in principle, an exact account of exchange effects for surfaces, i.e., the boundary between regions of higher and lower electron densities, whereas for internal regions this surface-exchange description is merged with that of the LDA. It relies on reference input other than formal manyparticle theory, and it is not usually counted as constraintbased [54,66], yet it is parameter-free and therefore included in this benchmarking. Like PBEsol, the AM05 functional extracts the gradient-corrected correlation from a study of the jellium surface energy. While it has different roots from those of PBE and PBEsol, it can be viewed as a semilocal functional since it is possible to express the energy per particle variation using Eq. (1). Like the regular GGAs, it lacks an account of truly nonlocal correlation effects. The exchange enhancement in AM05 is such that the 1-LOB condition (2) can be broken, but it is not clear to what extend it will affect calculations for dense matter, e.g., transition metals and other bulk systems.

\section{E. The vdW-DF framework}

The vdW-DF method $[6-8,10,12,16]$ represents a systematic nonempirical extension of both LDA and the semilocal GGA description $[9,13,21]$. The very first version of this method was conceived two decades ago starting from a simple Ashcroft picture [11] of vdW binding in the itinerant electron gas $[10,13]$. It provides seamless integration with a GGA-type description while enforcing conservation laws on the underlying many-body response description $[7,8,10,13]$. The method predates the PBEsol and AM05 functionals, and its origin [12] actually coincides with the launching of the PBE functional [4]. The vdW-DF method captures

approximations for the semilocal $\mathrm{XC}$ hole. In the case of the $\mathrm{PBE}$ functional, one arrives at a small-s expansion that is also suggested by exact-scaling results for atomiclike high-density regions $[5,54,71]$. By contrast, in the construction of PBEsol, one obtains a behavior consistent with diagrammatic results for pure exchange in the weakly perturbed electron gas. 
$\mathrm{vdW}$ forces among dimers in the asymptotic and the binding limits [7,72-77] as well as attraction between two-dimensional layers $[6,41-43,68]$. Importantly, it also captures the more general problem when nonlocal correlation forces compete with other types of forces [9], for example, giving rise to binding across important regions of low electron densities $[10,14]$. The method was expected to be relevant in first-principles DFT for both pure vdW problems including regular physisorption [49,78-80], porous-materials gas absorption [81-90], and DNA base-pair interactions [91-95]. It was also quickly realized that truly nonlocal correlations affect materials descriptions much more broadly than what was perhaps originally anticipated $[10,14,57,77,96-100]$. The recent vdWDF formulations are, for example, proving themselves valuable in the treatment of organic-inorganic interfaces and general weak-chemisorption problems [18,21,35,49,50,96,101-104].

The vdW-DF framework formally constitutes an ACF recast $[7,10,13]$

$$
E_{\mathrm{xc}}=\int_{0}^{\infty} \frac{d u}{2 \pi} \operatorname{Tr}\{\ln [\nabla \varepsilon(i u) \cdot \nabla G]\}-E_{\text {self }} .
$$

Here, $E_{\text {self }}$ and $G$ denote the Coulomb self-energy and Green function, respectively, $u$ is a complex frequency, and $\varepsilon$ denotes a suitable approximation for a scalar, nonlocal dielectric function. The trace is over all spatial coordinates. The $\mathrm{vdW}$-DF framework has exact screening, and it defines $\varepsilon$ via a plasmon-pole response description that reflects constraints such as the $F$ sum rule $[7,9,10,13]$. The vdW-DF method begins with a plasmon-pole approximation for a screened response treated at the GGA level, i.e., by choosing $\varepsilon(i u)$ so that it reflects the shape of an internal XC hole corresponding to a GGA-type semilocal functional $E_{\mathrm{xc}}^{\mathrm{in}}$. The vdW-DF method proceeds to define semilocal and nonlocal functional components,

$$
E_{\mathrm{xc}}^{\mathrm{vdW}-\mathrm{DF}}=E_{\mathrm{xc}}^{0}+E_{c}^{\mathrm{nl}},
$$

where the semilocal component satisfies

$$
E_{\mathrm{xc}}^{0} \approx E_{\mathrm{xc}}^{\mathrm{in}}
$$

while the truly nonlocal XC energy term is defined as

$$
E_{c}^{\mathrm{nl}}=\int_{0}^{\infty} \frac{d u}{2 \pi} \operatorname{Tr}\{\ln [\nabla \varepsilon(i u) \cdot \nabla G]-\ln [\varepsilon(i u)]\} .
$$

The vdW-DF framework can be interpreted as a rigorous implementation of the Ashcroft picture of $\mathrm{vdW}$ forces since Eq. (6) formally counts the shifts in electronic zero-point energies that arise with an electrodynamical coupling between the internal GGA-type XC holes [13]. In the most widely used general-geometry versions, the evaluation of Eq. (6) involves a second-order expansion that allows an efficient universal kernel formulation $[7,8,10]$. The vdW-DF versions are entirely nonempirical and rest solely on the physics that underpins the LDA XC energy and the GGA-type gradient-corrected exchange in $E_{\mathrm{xc}}^{0}$ and $E_{\mathrm{xc}}^{\mathrm{in}}$.

Here we benchmark the finite-temperature performance of the recent consistent-exchange version vdW-DF-cx [16]. In this functional, the exchange component in $E_{\mathrm{xc}}^{0}$ is chosen to minimize

$$
\delta E_{x}^{0} \equiv E_{\mathrm{xc}}^{0}-E_{\mathrm{xc}}^{\mathrm{in}}
$$

for small-to-medium values of the scaled density gradient $s$. In practice, this means that $\Delta E_{x}^{0}=0$ is for all systems but atoms and small molecules $[10,13,16]$ so that vdW-DF-cx effectively serves as an implementation of (an expanded form of) the full vdW-DF framework Eq. (3) [7,9,10,13]. Additional documentation for this new vdW-DF version can be found in Refs. [9,10,13].

In the design of vdW-DF2 and vdW-DF-cx, compliance with criteria (4) was prioritized over having compliance with the 1-LOB condition (2), and hence an automatic, universal compliance with the g-LOB criteria (3). For the present focus on materials with dense electron distributions and metallic bonding, the 1-LOB condition is not expected to be broken at any relevant spatial points. We also note that Refs. $[16,105]$ show that the vdW binding among molecular fragments almost always arises from regions with small to moderate values of $s>2-3$. There is no breaking of even the l-LOB condition from such regions (either) when using vdW-DF-cx.

\section{METHODOLOGY}

\section{A. Computational details}

DFT calculations were carried out using the projector augmented wave (PAW) method [106] as implemented in the Vienna ab-initio simulation package (VASP) [107]. For vdWDF-cx calculations, we used the patch released in Ref. [41]. In primitive cell calculations, the Brillouin zone was sampled using $\Gamma$-centered $\boldsymbol{k}$-point grids with $13 \times 13 \times 7$ divisions for hexagonal-close-packed (hcp) structures, $14 \times 14 \times 14$ divisions for face-centered-cubic (fcc) structures, and $15 \times 15 \times 15$ divisions for body-centered-cubic (bcc) structures. The planewave cutoff energy was chosen $30 \%$ larger than the commonly recommended value for each element in order to obtain very well converged forces and especially stresses. The values employed are tabulated in Table $\mathrm{V}$ of the supplementary material, which also provides details concerning the PAW setups. Generally, we employ PAW setups from the 2012 VASP database with the exception of $\mathrm{W}$, for which we consider a semicore setup that includes the $5 p$ (but not the $5 s$ states) in the valence, the performance of which has been carefully assessed previously [108,109]. These PAW setups have been shown to yield very good agreement with respect to all-electron calculations [110,111].

\section{B. Vibrational modeling}

To evaluate finite-temperature properties, we employed the quasiharmonic approximation (QHA). First, the harmonic Helmholtz free energy $F(T, V)$ was evaluated as a function of temperature at a fixed volume $V$ according to $[112,113]$

$$
F=\frac{1}{2} \sum_{\boldsymbol{q} \nu} \hbar \omega_{\boldsymbol{q} \nu}+k_{B} T \sum_{\boldsymbol{q} \nu} \ln \left[1-\exp \left(-\hbar \omega_{\boldsymbol{q} v} / k_{B} T\right)\right] .
$$

Here, the summations are the result of a discretization of the integral over the vibrational density of states and carried out over phonon modes with momentum $\hbar \boldsymbol{q}$ and index $v$. The Gibbs free energy $G(T, p)$ at constant pressure $p$ is obtained by repeating the calculation of $F(T, V)$ for a range of volumes and minimizing the sum of internal energy $U(V)$, Helmholtz 
energy $F(T, V)$, and the pressure-volume term according to

$$
G(T, p)=\min _{V}[U(V)+F(T, V)+p V] .
$$

While the internal energy $U(V)$ simply corresponds here to the Born-Oppenheimer energy as a function of volume, evaluation of the vibrational contribution Eq. (8) requires knowledge of the phonon dispersion on a dense $\boldsymbol{q}$-point mesh. Toward that end, force constants were calculated using the finite displacement method and $4 \times 4 \times 4$ supercells. In the latter calculations, the Brillouin zone was sampled using $\Gamma$-centered $3 \times 3 \times 3 \boldsymbol{k}$-point grids. The minimization in Eq. (9) was carried out over volumes ranging from $0.85 V_{0}$ to $1.15 V_{0}$, where $V_{0}$ is the volume corresponding to the minimum of the Born-Oppenheimer energy landscape.

Knowledge of the Gibbs free energy as a function of volume and temperature allows one to readily extract, for example, the lattice parameter(s), the bulk modulus, and the thermal expansion coefficient(s) at finite temperatures. All of these thermophysical properties were extracted using the PHONOPY package [113]. Specifically, the bulk modulus was obtained by fitting the Gibbs free energy to the Vinet equation of state [114].

Furthermore, we calculated the cohesive energy $E_{\text {coh }}$ at 0 $\mathrm{K}$ including the zero-point energy (ZPE) contribution,

$$
E_{\mathrm{coh}}=E_{\mathrm{bulk}}+\frac{1}{2} \sum_{\boldsymbol{q} v} \hbar \omega_{\boldsymbol{q} v}-E_{\mathrm{atom}},
$$

where $E_{\text {bulk }}$ and $E_{\text {atom }}$ denote the total energy of the bulk material and the atom, respectively. All terms in the latter equation were evaluated at the $0 \mathrm{~K}$ lattice constant corrected for zero-point effects.

\section{Atomic reference energies: Spin effects}

In general, spin polarization must be included when calculating $E_{\text {atom }}$. While a consistent spin-polarized version of the vdW-DF method was recently introduced [21], it has so far only been implemented in the QUANTUM-ESPRESSO package [115]. The vdW-DF evaluation of nonlocal correlations amounts to tracking the total energy shift that arises with the electrodynamical coupling of plasmons, which, in turn, represent a GGA-type response to external fields [13]. The vdW-DF approximations that are implemented in VASP [19] are not fully consistent since they ignore the fact that spin polarization will itself adjust these plasmons [21].

In the present study, we therefore proceeded as follows in order to obtain atomic reference energies and eventually cohesive energies for vdW-DF-cx. We calculated the nonspin-polarized atomic energy $E_{\text {atom }}^{\text {nsp, vasp }}$ using VASP and then added the atomic spin-polarization energy $\Delta_{\text {spin }}^{\mathrm{qe}}$ obtained using QUANTUM-ESPRESSO with the rigorous-spin vdW-DF-cx description [21]. That is, we obtained the atomic energies as

$$
E_{\text {atom }}=E_{\text {atom }}^{\mathrm{nsp}, \text { vasp }}+\underbrace{E_{\text {atom }}^{\mathrm{sp}, \mathrm{qe}}-E_{\text {atom }}^{\mathrm{nsp}, \mathrm{qe}}}_{\Delta_{\text {spin }}^{\mathrm{qe}}} .
$$

In effect, this procedure amounts to computing VASP and QHAbased cohesive-energy estimates $E_{\text {coh }}^{\text {vasp }}$ and then adding a spin correction

$$
\Delta_{\text {spin-correction }}=\Delta_{\text {spin }}^{\mathrm{qe}}-\Delta_{\text {spin }}^{\mathrm{vasp}},
$$

where $\Delta_{\text {spin }}^{\text {vasp }}=E_{\text {atom }}^{\mathrm{sp} \text {, vasp }}-E_{\text {atom }}^{\mathrm{nsp}, \mathrm{qe}}$ represents the VASP approximation for the vdW-DF-cx atomic spin-polarization energy. A detailed compilation of the atomic reference energies can be found in Table VI of the supplementary material.

In the QUANTUM-ESPRESSO calculations of $\Delta_{\text {spin }}$, we relied on norm-conserving pseudopotentials (NCPP) from the ABINIT package [116], using a plane-wave (density) cutoff of $80 \mathrm{Ry}$ (400 Ry) so as to best mimic the fact the VASP calculations are based on hard PAW setups. This NCPP choice was possible for all but the case of $\mathrm{W}$, where the ABINIT NCPP did not yield the correct spin-polarization state. For the $\mathrm{W}$ case alone, we therefore relied on a $\mathrm{W}$ ultrasoft pseudopotential in calculating $\Delta_{\text {spin correction. }}{ }^{2}$

\section{RESULTS}

\section{A. General assessment}

In this section, we provide an overview of the key results from our comparative analysis of constraint-based $\mathrm{XC}$ functionals. A complete compilation of the data obtained with each XC functional, including lattice constants, can be found in the supplementary material. To measure and compare the performance of different functionals, we consider the mean absolute percentage error (MAPE) defined as

$$
M=\frac{1}{N} \sum_{k}\left|\frac{A_{\mathrm{DFT}}^{(k)}-A_{\mathrm{expt}}^{(k)}}{A_{\mathrm{expt}}^{(k)}}\right|,
$$

where $A_{\mathrm{DFT}}^{(k)}$ and $A_{\text {ref }}^{(k)}$ denote predicted and experimental values of a property of structure $k$, and the average contains $N$ samples.

Many properties exhibit characteristic variations across the transition-metal series, which follow the $d$-band filling (Fig. 1) and are reproduced by all $\mathrm{XC}$ functional considered here. While ZPE and thermal expansion effects are generally limited to a few percent of the volume, they are nonetheless crucial for an accurate assessment.

The performance comparison (Fig. 2 and Table I) confirms that PBEsol and, with the exception of the cohesive energy, also AM05 represent general improvements over PBE. The relatively large MAPEs (Table I) arise mostly from larger errors in just a few systems. In the case of PBE, the MAPE for the cohesive energy of bcc structures is particularly large. This issue is primarily caused by an inaccurate description of the electronic configuration of the isolated spin-polarized atoms, which impacts the atomic reference energy.

More interestingly, the comparison demonstrates that the truly nonlocal vdW-DF-cx performs at least at the level of PBEsol and AM05. In fact, considering all the properties, vdW-DF-cx provides the best overall agreement with the experimental reference data. This is especially remarkable since previous nonempirical versions of the DF method, namely vdW-DF1 [7] (in which exchange is approximated

\footnotetext{
${ }^{2}$ We also tested using the W NCPP while constraining the spin polarization to the correct configuration, which yields a vdW-DF-cx value for the cohesive energy of $\mathrm{W}$ that is in even better agreement with experiment than when using the ultrasoft pseudopotential.
} 

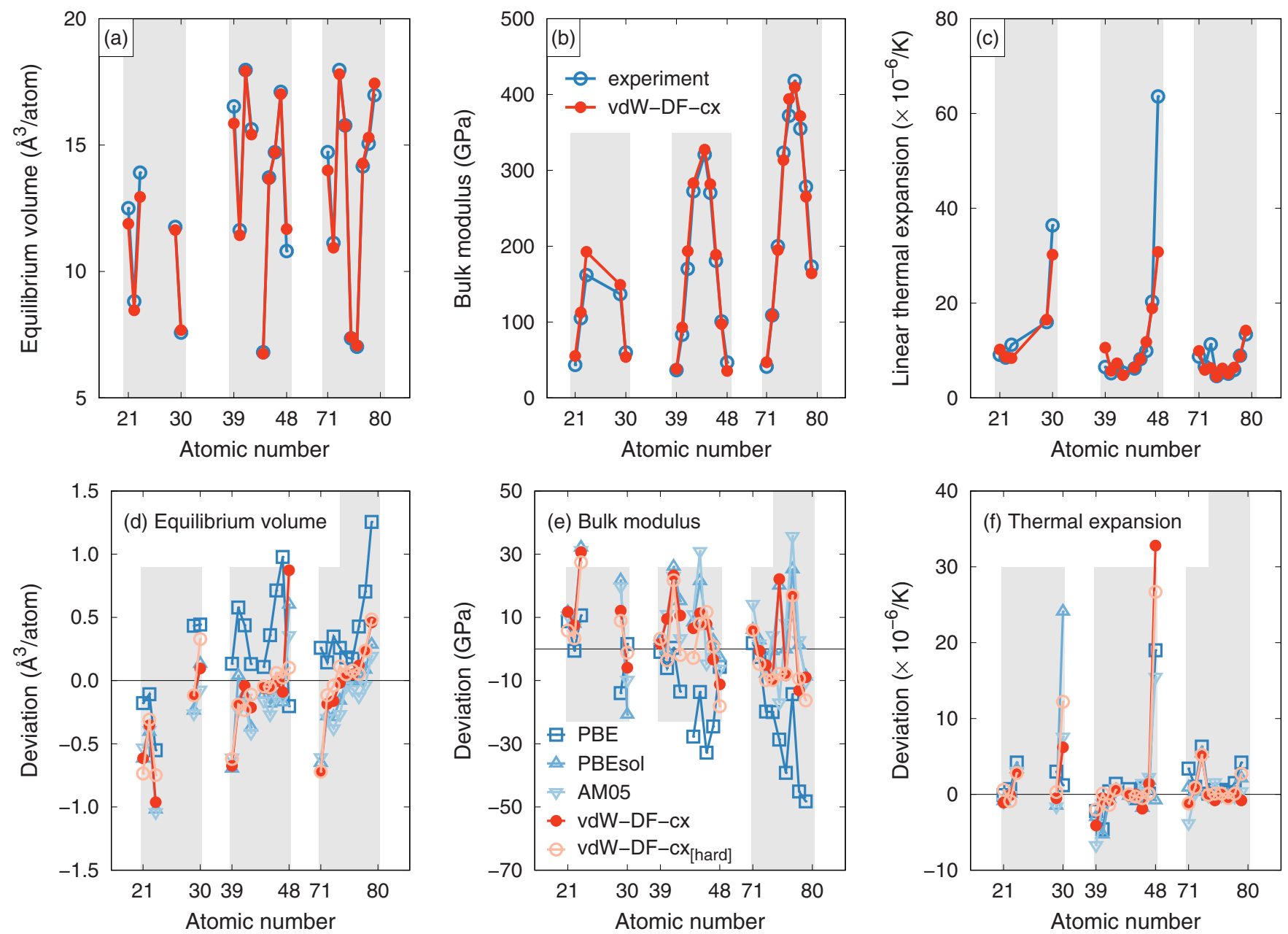

FIG. 1. Overview of thermophysical properties at $300 \mathrm{~K}$ obtained using the quasiharmonic approximation in conjunction with densityfunctional-theory calculations. (a) Equilibrium volume, (b) bulk modulus, and (c) coefficient of average linear thermal expansion from experiment (Refs. [52,53]) and calculations based on the vdW-DF-cx functional. Deviation between different XC functionals and experiment for (d) equilibrium volume, (e) bulk modulus, and (f) coefficient of linear thermal expansion. The shaded regions indicate the set of $3 d$, $4 d$, and $5 d$ transition metals.

by the revPBE functional [117]) and vdW-DF2 [15] (in which exchange is approximated by a revised version [69] of the PW86 functional [70]), perform rather poorly for the late transition metals. In particular, in the case of $\mathrm{Ag}$ and $\mathrm{Au}$, the lattice constants are considerably overestimated in vdW-DF1 and vdW-DF2 [19], while vdW-DF-cx yields excellent results for these elements.

\section{B. Cohesive energies}

Overall the constraint-based functionals considered here perform reasonably well with regard to the description of the cohesive energy (Fig. 3), although for most of the functionals there are problems with specific elements. Most notably, the vdW-DF-cx description clearly outperforms the other functionals in terms of the cohesive energies.

Moreover, the results demonstrate that the rigorous inclusion of spin effects in vdW-DF-cx [21] is important for an accurate description of the cohesive energy in nonmagnetic transition metals [Fig. 3(c)]. Since the atomic spin-polarization energies are very large in the middle of the transition-metal bands [Fig. 3(d)], it is important to use the rigorous-spin vdW-DF-cx formulation [21]. The corrections are negative and systematically lead to larger values for $\Delta_{\text {spin }}$ (see Table VI of the supplementary material). As a consequence, our rigorous-spin vdW-DF-cx calculations provide a systematic improvement for the description of nonmagnetic transition metals, lowering, for example, the MAPE from $9.1 \%$ to $7.3 \%$ when using hard PAW setups for vdW-DF-cx.

\section{Effect of PAW setups in the case of vdW-DF-cx}

For computational efficiency, it is often desirable to employ PAW setups that contain only the highest occupied states in the valence. This not only limits the total number of states in the calculation, but it also often allows using relatively large core radii that require smaller plane-wave-basis cutoffs in order to obtain converged results. While so far we have only considered results obtained using such "standard" PAW setups, it is now instructive to examine the choice of the PAW setup more closely. Toward that end, we exclusively consider calculations 

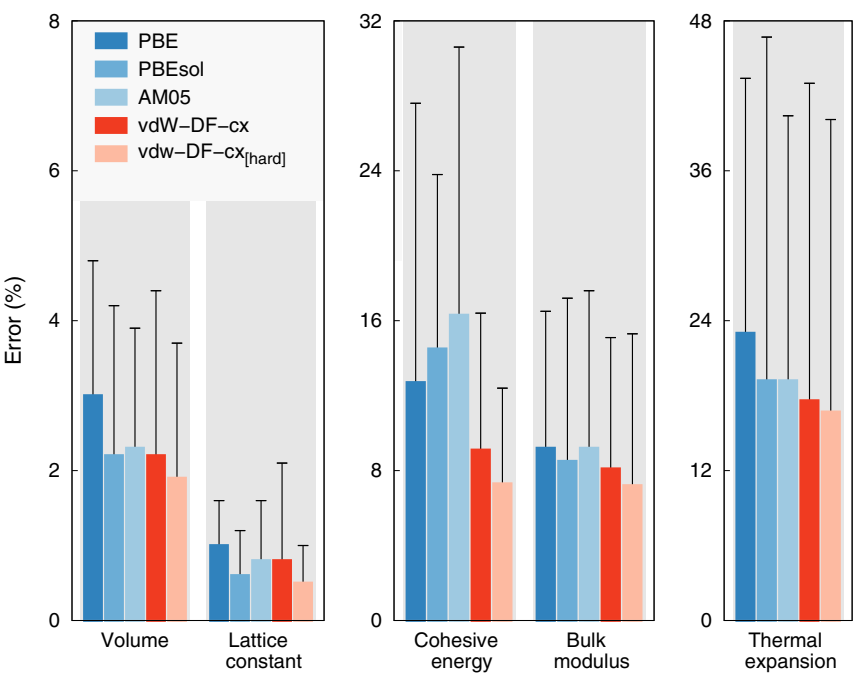

FIG. 2. Performance of different constraint-based XC functionals based on the data from Table I. The MAPEs were computed with regard to thermophysical properties measured at $300 \mathrm{~K}$, with the exception of the cohesive energy, for which $0 \mathrm{~K}$ values are compared. Standard deviations of the distribution of errors for each property and functionals are shown as black error bars.

based on the vdW-DF-cx functional and "hard" PAW setups, as detailed in Table $\mathrm{V}$ of the supplementary material.

Using the hard PAW setups systematically improves the agreement with experiment, typically reducing the MAPE by a fraction of a percent (Table I and Fig. 2). Yet, the comparison clearly demonstrates that already the "standard" PAW setups yield very good result and are sufficient to achieve good results in many situations. It should be noted that the comparison here is restricted to the vdW-DF-cx method, but a similar improvement when moving from standard to hard setups is likely to be observable also for the other functionals.

Cadmium represents an exception, for which there is a pronounced difference between standard and hard setups. For example, the lattice constants at $300 \mathrm{~K}$ change from $a=3.168 \AA$ and $c=5.373 \AA$ to $a=3.023 \AA$ and $c=5.512 \AA$ when going from standard to hard PAW setups. The latter values are also in notably better agreement with the experimental numbers of $a=2.98 \AA$ and $c=5.62 \AA$ (Table II of the supplementary material). More generally, the late transition metals in hcp structure ( $\mathrm{Zn}$ and $\mathrm{Cd}$ ) are challenging for all XC functionals. This behavior is related to their special electronic structure, which manifests itself, e.g., in $c / a$ ratios (experimentally $c / a=1.89$ and 1.86 for $\mathrm{Cd}$ and $\mathrm{Zn}$, respectively) that are considerably larger than in the ideal hcp structure $(c / a=1.633)$.

\section{Structure trends, semilocal, and nonlocal functionals}

The deviations between calculated and experimental data follow certain trends [Figs. 1(d)-1(f)]. While PBE tends to overestimate the equilibrium volume, the other functionals are overall in rather close agreement with the reference data.

Contrasting specifically the vdW-DF-cx and PBEsol performance (Fig. 4) shows that both functionals exhibit similar trends with respect to the variation of the accuracy with $d$-band filling. It is apparent that the data for the first and last
TABLE I. Performance of constraint-based XC functionals with respect to the description of thermophysical properties. The comparison includes the equilibrium volume $V$, bulk modulus $B$, as well as the linear coefficient of thermal expansion $\alpha_{l}$ measured at room temperature, while in the case of the cohesive energy $E_{\text {coh }}$ zero Kelvin data are compared, albeit including ZPE effects. Unless otherwise noted, the calculations were carried out using standard PAW setups. The comparison comprises $11 \mathrm{hcp}, 7 \mathrm{fcc}$, and 5 bcc elements.

\begin{tabular}{llcrrr}
\hline \hline Functional & & $V$ & $B$ & \multicolumn{1}{c}{$\alpha_{l}$} & \multicolumn{1}{c}{$E_{\text {coh }}$} \\
\hline vdW-DF-cx & hcp & $3.0 \%$ & $9.9 \%$ & $20.6 \%$ & $10.9 \%$ \\
& fcc & $1.0 \%$ & $5.0 \%$ & $5.9 \%$ & $9.5 \%$ \\
& bcc & $1.9 \%$ & $8.4 \%$ & $27.3 \%$ & $4.5 \%$ \\
& total & $2.2 \%$ & $8.1 \%$ & $17.6 \%$ & $9.1 \%$ \\
vdW-DF-cx & hcp & $2.6 \%$ & $8.5 \%$ & $17.7 \%$ & $7.8 \%$ \\
(hard PAW & fcc & $1.0 \%$ & $4.9 \%$ & $5.5 \%$ & $8.7 \%$ \\
setups) & bcc & $1.7 \%$ & $7.7 \%$ & $30.0 \%$ & $4.2 \%$ \\
& total & $1.9 \%$ & $7.2 \%$ & $16.7 \%$ & $7.3 \%$ \\
PBE & hcp & $2.3 \%$ & $7.1 \%$ & $23.6 \%$ & $10.9 \%$ \\
& fcc & $4.6 \%$ & $15.1 \%$ & $11.8 \%$ & $8.1 \%$ \\
& bcc & $2.2 \%$ & $5.6 \%$ & $37.4 \%$ & $23.3 \%$ \\
& total & $3.0 \%$ & $9.2 \%$ & $23.0 \%$ & $12.7 \%$ \\
PBEsol & hcp & $2.8 \%$ & $9.9 \%$ & $21.7 \%$ & $14.7 \%$ \\
& fcc & $1.1 \%$ & $6.1 \%$ & $8.4 \%$ & $12.4 \%$ \\
& bcc & $2.6 \%$ & $9.0 \%$ & $28.7 \%$ & $16.8 \%$ \\
& total & $2.2 \%$ & $8.5 \%$ & $19.2 \%$ & $14.5 \%$ \\
AM05 & hcp & $2.7 \%$ & $11.3 \%$ & $22.3 \%$ & $25.7 \%$ \\
& fcc & $1.2 \%$ & $6.8 \%$ & $6.8 \%$ & $9.7 \%$ \\
& bcc & $3.0 \%$ & $7.8 \%$ & $29.8 \%$ & $4.8 \%$ \\
& total & $2.3 \%$ & $9.2 \%$ & $19.2 \%$ & $16.3 \%$ \\
\hline \hline
\end{tabular}

columns of the series are slightly under- and overestimated, respectively. The largest relative corrections of the volume arise for $\mathrm{Zn}$ and $\mathrm{Cd}$, and those elements also have some of the largest vibrational corrections to the cohesive energies. In fact, most DFs provide an inaccurate description of these elements, which, as indicated above, exhibit a hcp structure with a very large axial ratio.

Larger deviations from the reference data are also observed for $\mathrm{V}$ (bcc) for all functionals. We ascribed this behavior to the low-temperature magnetism that has been reported in this element [118], while in the present calculations it is treated without spin polarization.

Similar trends as for the equilibrium volume can be observed for the bulk modulus [in reverse fashion, Fig. 1(e)] and the linear coefficient of thermal expansion [Fig. 1(f)], although the errors are more scattered. The latter effect is probably connected to a larger uncertainty in the experimental data, as will be discussed in the next section.

\section{E. Bulk modulus and thermal expansion}

So far we have used experimental values from compilations of standard values $[52,53]$ as reference data for equilibrium volumes (lattice constants), bulk moduli, and thermal expansion coefficients. While the data for lattice constants are usually very accurate, it must be acknowledged that measurements of bulk moduli and thermal expansion coefficients can carry rather significant errors, which are usually not documented in 

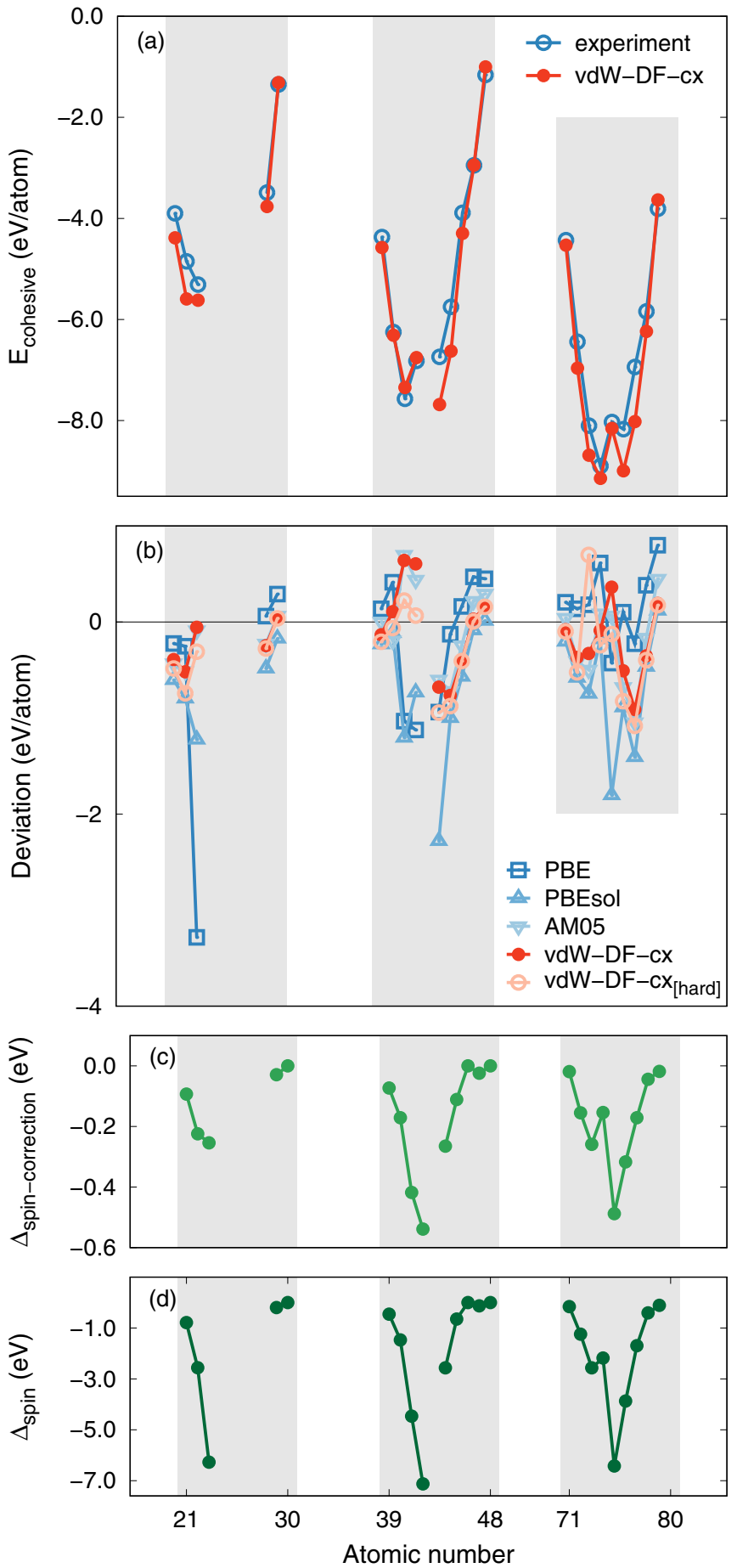

FIG. 3. (a) Cohesive energies at $0 \mathrm{~K}$ obtained using the quasiharmonic approximation in conjunction with density-functionaltheory calculations from experiment (Ref. [52]) and calculations based on the vdW-DF-cx functional including the spin correction according to Eq. (12). (b) Deviation between different XC functionals and experiment for cohesive energies. (c) Spin correction according to Eq. (12) that is added to the VASP data for our vdW-DF-cx benchmark to include a rigorous description of spin effects in the atomic reference energies. (d) Atomic spin polarization energies $\Delta_{\text {spin }}$ defined in Eq. (11) as calculated in the spin vdW-DF-cx formulation (available via QUANTUM-ESPRESSO calculations).
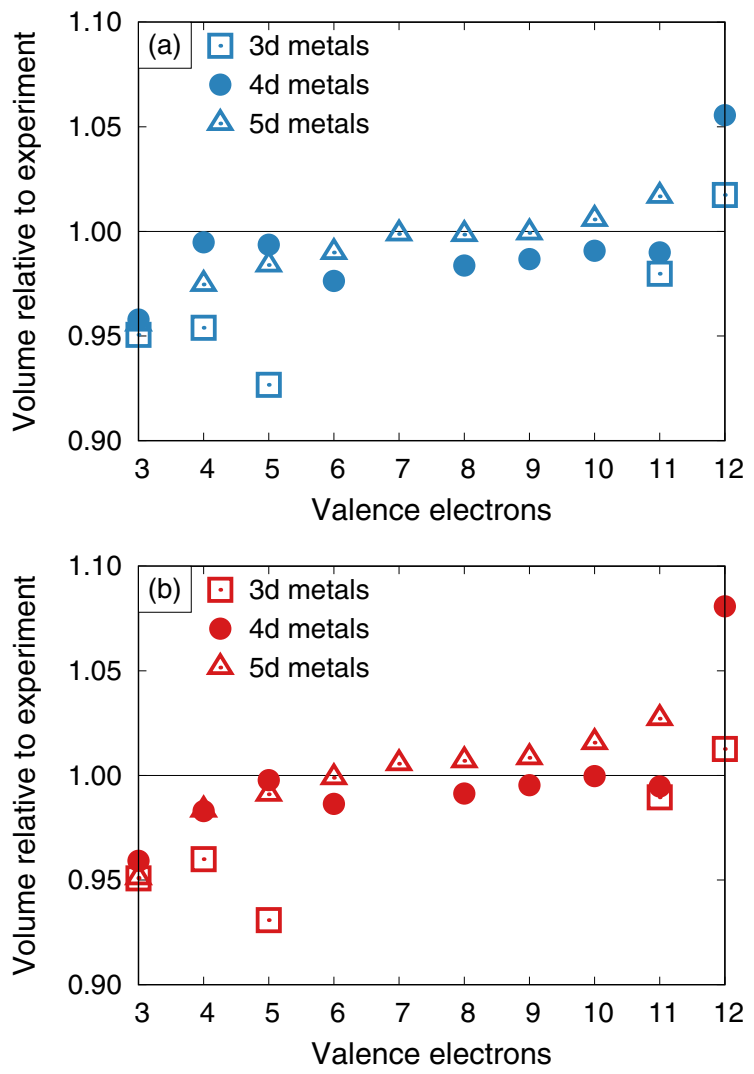

FIG. 4. Ratio of calculated to experimental equilibrium volumes for (a) PBEsol and (b) vdW-DF-cx (hard PAW setups). The transition from a volume underestimation converting to a volume overestimation is expected for GGAs and is found in both cases.

reference compendia. A closer inspection of experimental data available in the general scientific literature, however, reveals that at least in some cases these errors can be comparable to or even exceed the deviation between the best performing $\mathrm{XC}$ functionals and experiment.

For illustration purposes, we employ experimental data for the Young's moduli $E$ and Poisson ratios $v$ of polycrystalline samples of the fcc metals Ir, Pt, and Rh [119]. As the experimental data range from 300 to $1500 \mathrm{~K}$, this also allows us to compare experiment and calculations over a wide temperature span. The experimental data can be converted to the bulk modulus using the relation $B=E / 3(1-v)$. This illustrates that there is considerable scatter in the experimental data with changing temperature, which does not appear to be associated with a specific trend (Fig. 5); this is particularly pronounced in the case of Pt. The calculations overestimate the experimental data, but overall the agreement is good with a similar temperature dependence.

\section{F. General discussion}

High accuracy and transferability of vdW-DF-cx had been previously indicated by a range of successful applications to systems that combine regions of both sparse and dense electron distributions $[9,16,21,42,47-50]$. In the present paper, it has been demonstrated that, unlike the vdW-DF1 and 


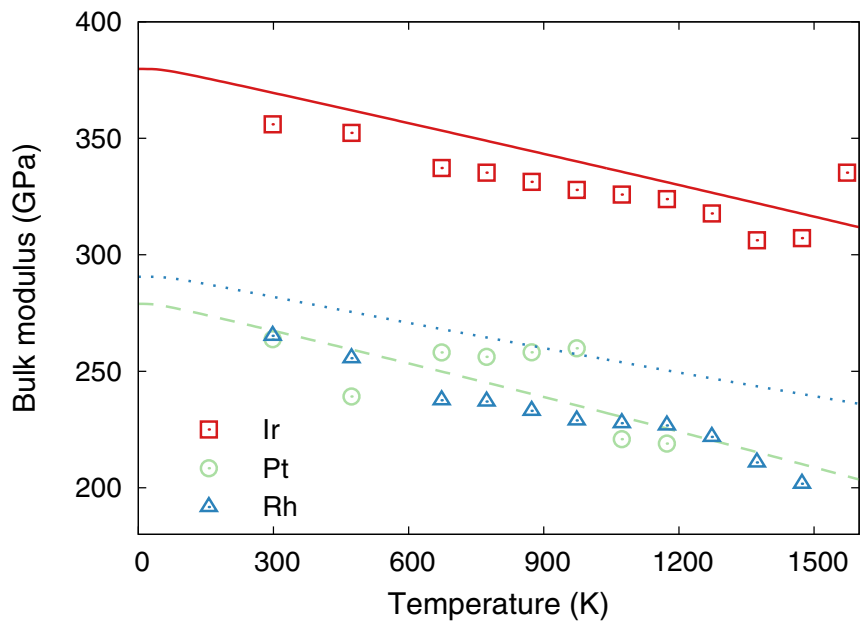

FIG. 5. Temperature dependence of the bulk modulus from experiment and calculation (vdW-DF-cx). The experimental data points were obtained by converting the data for Young's modulus $E$ and the Poisson ratio $v$ measured in Ref. [119] using the relation $B=E / 3(1-v)$.

vdW-DF2 versions, vdW-DF-cx also performs very well for hard materials.

The strong performance of PBEsol for traditional materials can be primarily traced to two of its features, namely a good form for gradient-corrected exchange and a good balance between this exchange part and the account of gradientcorrected correlation. The vdW-DF-cx strategy of seeking an ACF evaluation, see Eq. (3), implies picking a semilocal exchange component in $E_{\mathrm{xc}}^{0}$ that is given by a diagrammatic expansion and therefore is similar to that of PBEsol in the low-to-medium $s$ regime. Yet, vdW-DF-cx still replaces the PBEsol description of gradient-corrected correlation entirely with a truly nonlocal XC term $E_{c}^{\mathrm{nl}}$. The fact that DF-cx performs at a PBEsol level with respect to hard materials thus suggests that vdW-DF-cx achieves a good balance between exchange and correlation. This observation makes it plausible that one can obtain further functional improvements by relying on the ACF recast, Eq. (3), for descriptions of nonlocal correlation effects $[7,10,13,16]$.

\section{SUMMARY}

This study presents a comprehensive benchmark of constraint-based semilocal and nonlocal functionals with respect to finite-temperature thermophysical properties of nonmagnetic transition metals. The main outcome of this comparison is that, unlike its predecessors in the vdW-DF family, the recently developed nonlocal vdW-DF-cx version achieves good transferability and accuracy also for hard materials. This is crucial, for example, for investigations of weakly bound molecules at transition-metal surfaces. In the case of vdW-DF1 and vdW-DF2, the substantial overestimation of the lattice constants of the late transition metals, in particular Ag and $\mathrm{Au}$, limited their application to these systems. The successful validation of vdW-DF-cx for these cases allows full ionic relaxation and thus tracking of the impact of, e.g., associated adsorption-induced surface modifications $[9,19,50]$.

We note that Ambrosetti and Silvestrelli [39] recently presented a related benchmarking of several functionals, including vdW-DF-cx, for the coinage metals. Our findings are consistent with their results.

An excellent performance is expected for the other functionals considered here, but it is interesting to note that the truly nonlocal functional vdW-DF-cx has as good a performance and transferability, if not better. This is encouraging for further development that will build upon the vdW-DF framework.

Finally, we observe that quantitative comparisons, as shown in the tables included in the supplementary material, can also assist a crude benchmarking of future vDW-DF versions. This is because the tables implicitly provide a quantification of the net differences between the raw Kohn-Sham results and the associated room-temperature characterizations that are relevant for comparison with most experimental observations.

\section{ACKNOWLEDGMENTS}

We thank Kristian Berland for discussions. This work has been supported by the Swedish Research council (VR) and the Knut and Alice Wallenberg Foundation as well as computer time allocations by the Swedish National Infrastructure for Computing at NSC (Linköping), HPC2N (Umeå), and C3SE (Gothenburg).
[1] J. Harris and R. O. Jones, J. Phys. F 4, 1170 (1974).

[2] O. Gunnarsson and B. I. Lundqvist, Phys. Rev. B 13, 4274 (1976).

[3] D. C. Langreth and J. P. Perdew, Phys. Rev. B 15, 2884 (1977).

[4] J. P. Perdew, K. Burke, and M. Ernzerhof, Phys. Rev. Lett. 77, 3865 (1996).

[5] K. Burke, J. Chem. Phys. 136, 150901 (2012).

[6] H. Rydberg, M. Dion, N. Jacobson, E. Schröder, P. Hyldgaard, S. I. Simak, D. C. Langreth, and B. I. Lundqvist, Phys. Rev. Lett. 91, 126402 (2003).

[7] M. Dion, H. Rydberg, E. Schröder, D. C. Langreth, and B. I. Lundqvist, Phys. Rev. Lett. 92, 246401 (2004).

[8] T. Thonhauser, V. R. Cooper, S. Li, A. Puzder, P. Hyldgaard, and D. C. Langreth, Phys. Rev. B 76, 125112 (2007).
[9] K. Berland, C. Arter, V. R. Cooper, K. Lee, B. I. Lundqvist, E. Schröder, T. Thonhauser, and P. Hyldgaard, J. Chem. Phys. 140, 18A539 (2014).

[10] K. Berland, V. R. Cooper, K. Lee, E. Schröder, T. Thonhauser, P. Hyldgaard, and B. I. Lundqvist, IOP Rep. Progr. Phys. 78, 066501 (2015).

[11] K. Rapcewicz and N. W. Ashcroft, Phys. Rev. B 44, 4032(R) (1991).

[12] Y. Andersson, D. C. Langreth, and B. I. Lundqvist, Phys. Rev. Lett. 76, 102 (1996).

[13] P. Hyldgaard, K. Berland, and E. Schröder, Phys. Rev. B 90, 075148 (2014).

[14] D. C. Langreth, B. I. Lundqvist, S. D. Chakarova-Käck, V. R. Cooper, M. Dion, P. Hyldgaard, A. Kelkkanen, J. Kleis, 
L. Kong, S. Li, P. G. Moses, E. Murray, A. Puzder, H. Rydberg, E. Schröder, and T. Thonhauser, J. Phys.: Condens. Matter 21, 084203 (2009).

[15] K. Lee, É. D. Murray, L. Kong, B. I. Lundqvist, and D. C. Langreth, Phys. Rev. B 82, 081101 (2010).

[16] K. Berland and P. Hyldgaard, Phys. Rev. B 89, 035412 (2014).

[17] V. R. Cooper, Phys. Rev. B 81, 161104(R) (2010).

[18] J. Klimeš, D. R. Bowler, and A. Michaelides, J. Phys.: Condens. Matter 22, 022201 (2010).

[19] J. Klimeš, D. R. Bowler, and A. Michaelides, Phys. Rev. B 83, 195131 (2011)

[20] I. Hamada, Phys. Rev. B 89, 121103(R) (2014).

[21] T. Thonhauser, S. Zuluaga, C. A. Arter, K. Berland, E. Schröder, and P. Hyldgaard, Phys. Rev. Lett. 115, 136402 (2015).

[22] O. A. Vydrov and T. Van Voorhis, Phys. Rev. Lett. 103, 063004 (2009).

[23] O. A. Vydrov and T. Van Voorhis, J. Chem. Phys. 130, 104105 (2009).

[24] O. A. Vydrov and T. Van Voorhis, J. Chem. Phys. 133, 244103 (2010).

[25] A. D. Becke and E. R. Johnson, J. Chem. Phys. 127, 154108 (2007).

[26] A. D. Becke and E. R. Johnson, J. Chem. Phys. 123, 154101 (2005).

[27] A. D. Becke and E. R. Johnson, J. Chem. Phys. 122, 154104 (2005).

[28] S. Grimme, J. Comput. Chem. 27, 1787 (2006).

[29] A. Tkatchenko and M. Scheffler, Phys. Rev. Lett. 102, 073005 (2009).

[30] P. L. Silvestrelli, Phys. Rev. Lett. 100, 053002 (2008).

[31] P. L. Silvestrelli, J. Phys. Chem. A 113, 5224 (2009).

[32] S. Grimme, J. Antony, S. Ehrlich, and H. Krieg, J. Chem. Phys. 132, 154104 (2010).

[33] A. Ambrosetti and P. L. Silvestrelli, Phys. Rev. B 85, 073101 (2012).

[34] A. Tkatchenko, R. A. DiStasio, Jr., R. Car, and M. Scheffler, Phys. Rev. Lett. 108, 236402 (2012).

[35] V. G. Ruiz, W. Liu, E. Zojer, M. Scheffler, and A. Tkatchenko, Phys. Rev. Lett. 108, 146103 (2012).

[36] A. Ruzsinszky, J. P. Perdew, J. Tao, G. I. Csonka, and J. M. Pitarke, Phys. Rev. Lett. 109, 233203 (2012).

[37] J. Tao and J. P. Perdew, J. Chem. Phys. 141, 141101 (2014).

[38] J. Tao and A. M. Rappe, Phys. Rev. Lett. 112, 106101 (2014).

[39] A. Ambrosetti and P. L. Silvestrelli, Phys. Rev. B 94, 045124 (2016).

[40] P. Jurecka, J. Sponer, J. Cerny, and P. Hobza, Phys. Chem. Chem. Phys. 8, 1985 (2006).

[41] T. Björkman, J. Chem. Phys. 141, 074708 (2014).

[42] P. Erhart, P. Hyldgaard, and D. Lindroth, ACS Chem. Mater. 27, 5511 (2015).

[43] D. O. Lindroth and P. Erhart, Phys. Rev. B 94, 115205 (2016).

[44] M. Muruganathan, J. Sun, T. Imamura, and H. Mizuta, Nano Lett. 15, 8176 (2015).

[45] H. Sadeghi, S. Santarash, and C. J. Lambert, Sci. Rep. 5, 9514 (2015).
[46] M. Mehboudi, A. M. Dorio, W. Zhu, A. van der Zande, H. O. H. Churchill, A. A. Pacheco-Sanjuan, E. O. Harriss, P. Kumar, and S. Baraza-Lopez, Nano Lett. 16, 1704 (2016).

[47] T. Rangel, K. Berland, S. Sharifzadeh, F. Brown-Altvater, K. Lee, P. Hyldgaard, L. Kronik, and J. B. Neaton, Phys. Rev. B 93, 115206 (2016).

[48] F. Brown-Altvater, T. Rangel, and J. B. Neaton, Phys. Rev. B 93, 195206 (2016).

[49] J. Löfgren, H. Grönbeck, K. Moth-Poulsen, and P. Erhart, J. Phys. Chem. C 120, 12059 (2016).

[50] B. Borca, V. Schendel, R. Petuya, I. Pentegov, T. Michnowicz, U. Kraft, H. Klauk, A. Arnau, P. Wahl, U. Schlickum, and K. Kern, ACS Nano 9, 12506 (2015).

[51] N. Ashcroft and N. Mermin, Solid State Physics, Holt-Saunders International Editions: Science: Physics (Holt, Rinehart and Winston, Philadelphia, 1976).

[52] C. Kittel, Introduction to Solid State Physics (Wiley, New York, 1996).

[53] https://en.wikipedia.org/wiki/Thermal_expansion_coefficients _of_the_elements_(data_page).

[54] J. P. Perdew, A. Ruzsinszky, G. I. Csonka, O. A. Vydrov, G. E. Scuseria, L. A. Constantin, X. Zhou, and K. Burke, Phys. Rev. Lett. 100, 136406 (2008).

[55] R. Armiento and A. E. Mattsson, Phys. Rev. B 72, 085108 (2005).

[56] See Supplemental Material at http://link.aps.org/supplemental/ 10.1103/PhysRevB.95.085147 for a detailed quantitative presentation of $a b$ initio-based modeling and for a per element comparison with experimentally observed thermophysical properties. Tables I, II, and III provide a detailed comparison at $300 \mathrm{~K}$ for structural and thermophysical properties of $3 d, 4 d$, and $5 d$ nonmagnetic transition-metal elements, respectively. Table IV represents a comparison of cohesive energies, while Table V provides a specification of the PAW setups and the plane-wave energy cutoffs used in the calculations of different elements. Finally, Table VI summarizes and compares the terms that enter the computation of the cohesive energy in the case of vdW-DF-cx.

[57] E. Ziambaras, J. Kleis, E. Schröder, and P. Hyldgaard, Phys. Rev. B 76, 155425 (2007).

[58] J. Sun, B. Xiao, and A. Ruzsinszky, J. Chem. Phys. 137, 051101 (2012).

[59] J. Sun, A. Ruzsinszky, and J. P. Perdew, Phys. Rev. Lett. 115, 036402 (2015).

[60] J. Tao and Y. Mo, Phys. Rev. Lett. 117, 073001 (2016).

[61] D. C. Langreth and J. P. Perdew, Phys. Rev. B 21, 5469 (1980).

[62] D. C. Langreth and S. H. Vosko, Phys. Rev. Lett. 59, 497 (1987).

[63] J. P. Perdew and Y. Wang, Phys. Rev. B 46, 12947 (1992).

[64] L. Hedin and B. I. Lundqvist, J. Phys. C 4, 2064 (1971).

[65] E. H. Lieb and S. Oxford, Int. J. Quantum Chem. 19, 427 (1981).

[66] A. Ruzsinszky and J. P. Perdew, Comput. Theor. Chem. 963, 2 (2011).

[67] J. P. Perdew, A. Ruzsinszky, J. Sun, and K. Burke, J. Chem. Phys. 140, 18A533 (2014). 
[68] D. C. Langreth, M. Dion, H. Rydberg, E. Schröder, P. Hyldgaard, and B. I. Lundqvist, Int. J. Quantum Chem. 101, 599 (2005).

[69] E. D. Murray, K. Lee, and D. C. Langreth, J. Chem. Theor. Comput. 5, 2754 (2009).

[70] J. P. Perdew and Y. Wang, Phys. Rev. B 33, 8800 (1986).

[71] A. D. Becke, J. Chem. Phys. 140, 18A301 (2014).

[72] A. Puzder, M. Dion, and D. C. Langreth, J. Chem. Phys. 124, 164105 (2006).

[73] S. Li, V. R. Cooper, T. Thonhauser, A. Puzder, and D. C. Langreth, J. Phys. Chem. A 112, 9031 (2008).

[74] K. Berland and P. Hyldgaard, J. Chem. Phys. 132, 134705 (2010).

[75] K. Berland, Ø. Borck, and P. Hyldgaard, Comput. Phys. Commun. 182, 1800 (2011).

[76] M. Callsen and I. Hamada, Phys. Rev. B 91, 195103 (2015).

[77] O. A. Vydrov, and T. Van Voorhis, in Fundamentals of TimeDependent Density Functional Theory, edited by M. A. L. Marques, N. Maitra, F. Nogueira, E. K. U. Gross, and A. Rubio (Springer, Berlin, 2012).

[78] K. Lee, A. K. Kelkkanen, K. Berland, S. Andersson, D. C. Langreth, E. Schröder, B. I. Lundqvist, and P. P. Hyldgaard, Phys. Rev. B 84, 193408 (2011).

[79] K. Lee, K. Berland, M. Yoon, S. Andersson, E. Schröder, P. Hyldgaard, and B. I. Lundqvist, J. Phys.: Condens. Matter 24, 424213 (2012).

[80] E. Londero, E. K. Karlson, M. Landahl, D. Ostrovskii, J. D. Rydberg, and E. Schröder, J. Phys.: Condens. Matter 24, 424212 (2012).

[81] Y. Yao, N. Nijem, J. Li, Y. J. Chabal, D. C. Langreth, and T. Thonhauser, Phys. Rev. B 85, 064302 (2012).

[82] K. Tan, N. Nijem, P. Canepa, Q. Gong, J. Li, T. Thonhauser, and Y. J. Chabal, Chem. Mater. 24, 3153 (2012).

[83] R. Poloni, B. Smit, and J. B. Neaton, J. Phys. Chem. A 116, 4957 (2012).

[84] H. Liu, V. R. Cooper, S. Dai, and D.-e. Jiang, J. Phys. Chem. Lett. 3, 3343 (2012).

[85] K. Lee, W. C. Isley, A. L. Dzubak, P. Verma, S. J. Stoneburner, L.-C. Lin, J. D. Howe, E. D. Bloch, D. A. Reed, M. R. Hudson, C. M. Brown, J. R. Long, J. B. Neaton, B. Smit, C. J. Cramer, D. G. Truhlar, and L. Gagliardi, J. Am. Chem. Soc. 136, 698 (2014).

[86] R. Poloni, K. Lee, R. F. Berger, B. Smit, and J. B. Neaton, J. Phys. Chem. Lett. 5, 861 (2014).

[87] K. Tan, S. Zuluaga, Q. Gao, N. Nijem, J. Li, T. Thonhauser, and Y. J. Chabal, Chem. Mater. 27, 2203 (2015).

[88] S. Zuluaga, E. M. A. Fuentes-Fernandez, K. Tan, F. Xu, J. Li, Y. J. Chabal, and T. Thonhauser, J. Mater. Chem. A 4, 5176 (2016).

[89] R. Poloni and J. Kim, Int. J. Quantum Chem. 116, 569 (2016).

[90] E. Kuisma, C. F. Hansson, T. B. Lindberg, C. A. Gillberg, S. Idh, and E. Schröder, J. Chem. Phys. 144, 184704 (2016).

[91] F. Ortmann, W. G. Schmidt, and F. Bechstedt, Phys. Rev. Lett. 95, 186101 (2005).

[92] V. R. Cooper, T. Thonhauser, A. Puzder, E. Schröder, B. I. Lundqvist, and D. C. Langreth, J. Am. Chem. Soc. 130, 1304 (2007).
[93] S. Li, V. R. Cooper, T. Thonhauser, B. I. Lundqvist, and D. C. Langreth, J. Phys. Chem. B 113, 11166 (2009).

[94] K. Berland, S. D. Chakarova-Käck, V. R. Cooper, D. C. Langreth, and E. Schröder, J. Phys.: Condens. Matter 23, 135001 (2011).

[95] D. Le, A. Kara, E. Schröder, P. Hyldgaard, and T. S. Rahman, J. Phys.: Condens. Matter 24, 424210 (2012).

[96] S. D. Chakarova-Käck, O. Borck, E. Schröder, and B. I. Lundqvist, Phys. Rev. B 74, 155402 (2006).

[97] J. Kleis, E. Schröder, and P. Hyldgaard, Phys. Rev. B 77, 205422 (2008).

[98] K. Johnston, J. Kleis, B. I. Lundqvist, and R. M. Nieminen, Phys. Rev. B 77, 121404 (2008).

[99] K. Berland, T. L. Einstein, and P. Hyldgaard, Phys. Rev. B 80, 155431 (2009).

[100] J. Klimeš and A. Michaelides, J. Chem. Phys. 137, 120901 (2012).

[101] S. D. Chakarova-Käck, E. Schröder, B. I. Lundqvist, and D. C. Langreth, Phys. Rev. Lett. 96, 146107 (2006).

[102] G. Li, I. Tamblyn, V. R. Cooper, H.-J. Gao, and J. B. Neaton, Phys. Rev. B 85, 121409 (2012).

[103] J. Maurer, V. G. Ruiz, and A. Tkatchenko, J. Chem. Phys. 143, 102808 (2015).

[104] A. Tkatchenko, L. Romaner, O. T. Hofmann, E. Zojer, C. Ambrosch-Draxl, and M. Scheffler, MRS Bull. 35, 435 (2010).

[105] K. Berland and P. Hyldgaard, Phys. Rev. B 87, 205421 (2013).

[106] P. E. Blöchl, Phys. Rev. B 50, 17953 (1994); G. Kresse and D. Joubert, ibid. 59, 1758 (1999).

[107] G. Kresse and J. Hafner, Phys. Rev. B 47, 558 (1993); G. Kresse and J. Furthmüller, ibid. 54, 11169 (1996).

[108] L. Gharaee and P. Erhart, J. Nucl. Mater. 467, 448 (2015).

[109] L. Gharaee, J. Marian, and P. Erhart, J. Appl. Phys. 120, 025901 (2016).

[110] K. Lejaeghere, G. Bihlmayer, T. Björkman, P. Blaha, S. Blügel, V. Blum, D. Caliste, I. E. Castelli, S. J. Clark, A. D. Corso, S. d. Gironcoli, T. Deutsch, J. K. Dewhurst, I. D. Marco, C. Draxl, M. Dułak, O. Eriksson, J. A. Flores-Livas, K. F. Garrity, L. Genovese, P. Giannozzi, M. Giantomassi, S. Goedecker, X. Gonze, O. Grånäs, E. K. U. Gross, A. Gulans, F. Gygi, D. R. Hamann, P. J. Hasnip, N. A. W. Holzwarth, D. Iuşan, D. B. Jochym, F. Jollet, D. Jones, G. Kresse, K. Koepernik, E. Küçükbenli, Y. O. Kvashnin, I. L. M. Locht, S. Lubeck, M. Marsman, N. Marzari, U. Nitzsche, L. Nordström, T. Ozaki, L. Paulatto, C. J. Pickard, W. Poelmans, M. I. J. Probert, K. Refson, M. Richter, G.-M. Rignanese, S. Saha, M. Scheffler, M. Schlipf, K. Schwarz, S. Sharma, F. Tavazza, P. Thunström, A. Tkatchenko, M. Torrent, D. Vanderbilt, M. J. v. Setten, V. V. Speybroeck, J. M. Wills, J. R. Yates, G.-X. Zhang, and S. Cottenier, Science 351, aad3000 (2016).

[111] http://molmod.ugent.be/deltacodesdft.

[112] D. C. Wallace, Thermodynamics of Crystals (Dover, Mineola, NY, 1998).

[113] A. Togo and I. Tanaka, Scr. Mater. 108, 1 (2015).

[114] P. Vinet, J. R. Smith, J. Ferrante, and J. H. Rose, Phys. Rev. B 35, 1945 (1987).

[115] P. Giannozzi, S. Baroni, N. Bonini, M. Calandra, R. Car, C. Cavazzoni, D. Ceresoli, G. L. Chiarotti, M. Cococcioni, I. Dabo, A. D. Corso, S. de Gironcoli, S. Fabris, G. Fratesi, R. 
Gebauer, U. Gerstmann, C. Gougoussis, A. Kokalj, M. Lazzeri, L. Martin-Samos, N. Marzari, F. Mauri, R. Mazzarello, S. Paolini, A. Pasquarello, L. Paulatto, C. Sbraccia, S. Scandolo, G. Sclauzero, A. P. Seitsonen, A. Smogunov, P. Umari, and R. M. Wentzcovitch, J. Phys.: Condens. Matter 21, 395502 (2009).

[116] X. Gonze, G. M. Rignanese, M. Verstraete, J. M. Beuken, Y. Pouillon, R. Caracas, F. Jollet, M. Torrent, G. Zerah, M.
Mikami, P. Ghosez, M. Veithen, J. Y. Raty, V. Olevanov, F. Bruneval, L. Reining, R. Godby, G. Onida, D. R. Hamann, and D. C. Allan, Z. Kristall. 220, 558 (2005).

[117] Y. Zhang and W. Yang, Phys. Rev. Lett. 80, 890 (1998).

[118] Y. M. Smirnov and V. A. Finkel, Sov. Phys. JETP 22, 750 (1966).

[119] J. Merker, D. Lupton, M. Töpfer, and H. Knake, Platinum Met. Rev. 45, 74 (2001). 